Journal of Universal Mathematics

VOL.3 No.1 PP.21-27 (2020)

ISSN-2618-5660

\title{
BIPOLAR SOFT FILTER
}

\author{
ORHAN DALKILIÇ AND NAIME DEMIRTAŞ
}

\begin{abstract}
In this study, we present bipolar soft filters which are defined over an initial universe using a fixed parameter set. At the same time, the concepts of bipolar soft filter subbase and bipolar soft filter base are given. In addition, we give examples in order to better understand the subject in our paper.
\end{abstract}

\section{INTRODUCTION}

Mathematics is based on precise concepts and results. Therefore, mathematicians needed a model in case of uncertainty of concepts. The concept of soft sets was given by Molodtsov [7] as a approach in 1999 to model uncertainty. He [7] gave the basic results of this theory to solve many practical problems in economics, medical science, etc. Then Maji et al. [5] introduced some new concepts such as a subset, the complement of a soft set. Studies on soft sets are increasing rapidly in recent years.

Many researchers were studied the topological structure of soft sets: Shabir and Naz [9] introduced the soft topological spaces which are defined over an initial universe with a fixed set of parameters. They studied many concepts such as soft open set, soft neighbourhood of a point in soft topological spaces. As a different approach to soft topology; Cağman et al. [2] defined the concepts of soft open set, soft interior, soft closure, soft limit point, soft Hausdorff space. In addition, many researchers such as Aygünoğlu and Aygün [1], Min [6], Zorlutuna et al. [13], Hussain and Ahmad [3], Varol and Aygün [8] studied soft topological spaces.

In recent years, Shabir and Naz [10] and Karaaslan and Karatas [4] differently defined bipolar soft set. Clearly, bipolar soft sets achieved sharper results than soft sets and so the concept of bipolar soft topology is very important. Many researchers have conducted studies on bipolar soft topological space: Shabir M. and Bakhtawar A.[11] introduced the bipolar soft topological spaces. Then, Öztürk Y.T.[14] introduced some features of the bipolar soft topological space.

In this study, we introduce the bipolar soft filters established on the initial universe with a fixed set of parameters. To define the bipolar soft filters, we first gave

2000 Mathematics Subject Classification. 03E47; 54A05; 54C60.

Key words and phrases. Soft topological space, Bipolar soft topological space, Bipolar soft filter. 
the basic properties of the concepts of soft sets, soft topological spaces, bipolar soft sets, bipolar soft topological spaces. Also, we give bipolar soft filter subbase and bipolar soft filter base definitions by defining bipolar soft filter. Finally, we investigated some of the basic features of these defined concepts and supported with examples.

\section{Preliminaries}

In this section, we remember some fundamental concepts in soft sets, soft topological space, bipolar soft sets and bipolar soft topological space.

Let $X$ be an initial universe, and $E$ be a set of parameters. Let $P(X)$ denotes the power set of $X$ and $A, B$ be non-empty subsets of $E$.

Definition 2.1. [7] A pair $(F, A)$ is called a soft set over $U$, where $F$ is a mapping given by $F: A \rightarrow P(X)$.

Note that the set of all soft sets over $X$ will be denoted by $S(X, E)$.

Definition 2.2. [9] Let $\tilde{\tau} \sqsubseteq S(X, E)$. A soft topology on $X$, denoted by $\tilde{\tau}$, is a collection of soft subsets of $X$ having the following properties:

(1) $\Phi, \tilde{X} \in \tilde{\tau}$

(2) If $(F, E),(G, E) \in \tilde{\tau}$, then $(F, E) \cap(G, E) \in \tilde{\tau}$

(3) If $((F, E))_{\lambda} \in \tilde{\tau}, \forall \lambda \in \Delta$, then $\cup_{\lambda \in \Delta}((F, E))_{\lambda} \in \tilde{\tau}$

The pair $(X, \tilde{\tau})$ is called a soft topological space. Every member of $\tilde{\tau}$ is called a soft open set. A soft set $F_{A}$ is called soft closed in $(X, \tilde{\tau})$ if $F_{A}^{c} \in \tilde{\tau}$.

Definition 2.3. [12] Let $\tilde{\mathcal{F}} \sqsubseteq S(X, E)$ then $\tilde{\mathcal{F}}$ is a called a soft filter on $X$ if $\tilde{\mathcal{F}}$ satisfies the following properties:

$\left.f_{1}\right) \Phi \notin \tilde{\mathcal{F}}$,

$\left.f_{2}\right) \forall F_{A}, G_{B} \in \tilde{\mathcal{F}} \Longrightarrow F_{A} \cap G_{B} \in \tilde{\mathcal{F}}$

$\left.f_{3}\right) \forall F_{A} \in \tilde{\mathcal{F}}$ and $F_{A} \sqsubseteq G_{B} \Longrightarrow G_{B} \in \tilde{\mathcal{F}}$.

Now, we mention some basic concepts of bipolar soft sets and bipolar soft topological space.

Let $X$ be an initial universe, and $E$ be a set of parameters. Let $P(X)$ denotes the power set of $X$ and $A, B, C$ be non-empty subsets of $E$.

Definition 2.4. [5] Let $E=\left\{e_{1}, e_{2}, \ldots, e_{n}\right\}$ be a set of parameters. The NOT set of $E$ denoted by $\neg E$ is defined by $\neg E=\left\{\neg e_{1}, \neg e_{2}, \ldots, \neg e_{n}\right\}$ where, $\neg e_{i}=$ not $e_{i}$ for all $i$.

Definition 2.5. [10] A triplet $(F, G, A)$ is called a bipolar soft set over $X$, where $F$ and $G$ are mappings, given by $F: A \rightarrow P(X)$ and $G: \neg A \rightarrow P(X)$ such that $F(e) \cap G(\neg e)=\emptyset$ (Empty Set) for all $e \in A$.

Definition 2.6. [14] Let $(F, G, A)$ be a bipolar soft set over $X$. The presentation of

$(F, G, A)=\{(e, F(e), G(\neg e)): e \in A \subseteq E, \neg e \in \neg A \subseteq E$ and $F(e), G(\neg e) \in P(X)\}$

is said to be a short expansion of bipolar soft set $(F, G, A)$. 
Definition 2.7. [10] For two bipolar soft sets $(F, G, A)$ and $\left(F_{1}, G_{1}, B\right)$ over a universe $U$, we say that $(F, G, A)$ is a bipolar soft subset of $\left(F_{1}, G_{1}, B\right)$, if,

(1) $A \subseteq B$ and

(2) $F(e) \subseteq F_{1}(e)$ and $G_{1}(\neg e) \subseteq G(\neg e)$ for all $e \in A$.

This relationship is denoted by $(F, G, A) \tilde{\subseteq}\left(F_{1}, G_{1}, B\right)$. Similarly $(F, G, A)$ is said to be a bipolar soft superset of $\left(F_{1}, G_{1}, B\right)$, if $\left(F_{1}, G_{1}, B\right)$ is a bipolar soft subset of $(F, G, A)$. We denote it by $(F, G, A) \tilde{\supseteq}\left(F_{1}, G_{1}, B\right)$.

Definition 2.8. [10] Two bipolar soft sets $(F, G, A)$ and $\left(F_{1}, G_{1}, B\right)$ over a universe $U$ are said to be equal if $(F, G, A)$ is a bipolar soft subset of $\left(F_{1}, G_{1}, B\right)$ and $\left(F_{1}, G_{1}, B\right)$ is a bipolar soft subset of $(F, G, A)$.

Definition 2.9. [10] The complement of a bipolar soft set $(F, G, A)$ is denoted by $(F, G, A)^{c}$ and is defined by $(F, G, A)^{c}=\left(F^{c}, G^{c}, A\right)$ where $F^{c}$ and $G^{c}$ are mappings given by $F^{c}(e)=G(\neg e)$ and $G^{c}(\neg e)=F(e)$ for all $e \in A$.

Definition 2.10. [10] Bipolar soft union of two bipolar soft sets $(F, G, A)$ and $\left(F_{1}, G_{1}, B\right)$ over $X$ is the bipolar soft set $(H, I, C)$ over $U$, where $C=A \cup B$ and for all $e \in C$,

$$
\begin{gathered}
H(e)= \begin{cases}F(e) & \text { if } e \in A-B \\
F_{1}(e) & \text { if } e \in B-A \\
F(e) \cup F_{1}(e) & \text { if } e \in A \cap B\end{cases} \\
I(\neg e)= \begin{cases}G(\neg e) & \text { if } \neg e \in(\neg A)-(\neg B) \\
G_{1}(\neg e) & \text { if } \neg e \in(\neg B)-(\neg A) \\
G(\neg e) \cap G_{1}(\neg e) & \text { if } \neg e \in(\neg A) \cap(\neg B)\end{cases}
\end{gathered}
$$

We denote it by $(F, G, A) \tilde{\cup}\left(F_{1}, G_{1}, B\right)=(H, I, C)$.

Definition 2.11. [10] Bipolar soft intersection of two bipolar soft sets $(F, G, A)$ and $\left(F_{1}, G_{1}, B\right)$ over $X$ is the bipolar soft set $(H, I, C)$, over $X$ where $C=A \cap B$ is non-empty and for all $e \in C$,

$$
H(e)=F(e) \cap G(e) \text { and } I(\neg e)=F_{1}(\neg e) \cup G_{1}(\neg e)
$$

We denote it by $(F, G, A) \tilde{\cap}\left(F_{1}, G_{1}, B\right)=(H, I, C)$.

Definition 2.12. [10] Let $\left(F_{1}, G_{1}, A\right)$ and $\left(F_{2}, G_{2}, B\right)$ be two bipolar soft sets over $X$. Then,

(1) $\left(\left(F_{1}, G_{1}, A\right) \tilde{\cup}\left(F_{2}, G_{2}, B\right)\right)^{c}=\left(F_{1}, G_{1}, A\right)^{c} \tilde{\cap}\left(F_{2}, G_{2}, B\right)^{c}$

(2) $\left(\left(F_{1}, G_{1}, A\right) \tilde{\cap}\left(F_{2}, G_{2}, B\right)\right)^{c}=\left(F_{1}, G_{1}, A\right)^{c} \tilde{\cup}\left(F_{2}, G_{2}, B\right)^{c}$

Definition 2.13. [10] A bipolar soft set $(F, G, A)$ over $X$ is said to be relative null bipolar soft set, denoted by $(\Phi, \tilde{X}, A)$, if for all $e \in A, F(e)=\emptyset$ and for all $\neg e \in \neg A$, $G(\neg e)=X$. The relative null bipolar soft set with respect to the universe set of parameters $E$ is called a NULL bipolar soft set over $X$ and is denoted by $(\Phi, \tilde{X}, A)$.

Definition 2.14. [10] A bipolar soft set $(F, G, A)$ over $X$ is said to be relative absolute bipolar soft set, denoted by $(\tilde{X}, \Phi, A)$, if for all $e \in A, F(e)=X$ and for all $\neg e \in \neg A, G(\neg e)=\emptyset$. The relative absolute bipolar soft set with respect to the universe set of parameters $E$ is called a ABSOLUTE bipolar soft set over $X$ and is denoted by $(\tilde{X}, \Phi, E)$. 
Definition 2.15. [11] Let $\tilde{\tilde{\tau}}$ be the collection of bipolar soft sets over $X$ with $E$ as the set of parameters. Then $\tilde{\tilde{\tau}}$ is said to be a bipolar soft topology over $X$ if

(1) $(\Phi, \tilde{X}, A)$ and $(\tilde{X}, \Phi, A)$ belong to $\tilde{\tilde{\tau}}$

(2) the bipolar soft union of any number of bipolar soft sets in $\tilde{\tilde{\tau}}$ belong to $\tilde{\tilde{\tau}}$,

(3) the bipolar soft intersection of finite number of bipolar soft sets in $\tilde{\tilde{\tau}}$ belong to $\tilde{\tilde{\tau}}$.

Then $(X, \tilde{\tilde{\tau}}, E, \neg E)$ is called a bipolar soft topological space over $X$. the members of $\tilde{\tilde{\tau}}$ are said to be bipolar soft open sets in $X$. A bipolar soft set $(F, G, E)$ over $X$ is said to be a bipolar soft closed set in $X$, if its bipolar soft complemet $(F, G, E)^{c}$ belongs to $\tilde{\tilde{\tau}}$.

Definition 2.16. [11] Let $(X, \tilde{\tilde{\tau}}, E, \neg E)$ be a bipolar soft topological space over $X$. A bipolar soft set $(F, G, E)$ over $X$ is said to be a bipolar soft clopen set in $X$, if it is both a bipolar soft closed set and a bipolar soft open set over $X$.

\section{Bipolar Soft Filter}

From now on, all bipolar soft sets defined on parameter set $E$ and the universe sets $X$ represent by $B S(X, E, \neg E)$.

Definition 3.1. If $\widetilde{\mathcal{F G}} \sqsubseteq B S(X, E, \neg E)$ meets the following specifications, $\widetilde{\mathcal{F G}}$ is a defined a bipolar soft filter over $X$.

$\left.f g_{1}\right)(\Phi, \tilde{X}, E) \notin \widetilde{\mathcal{F} \mathcal{G}}$,

$\left.f g_{2}\right) \forall(F, G, E),\left(F_{1}, G_{1}, E\right) \in \widetilde{\mathcal{F G}} \Longrightarrow(F, G, E) \tilde{\cap}\left(F_{1}, G_{1}, E\right) \in \widetilde{\mathcal{F G}}$

$\left.f g_{3}\right) \forall(F, G, E) \in \widetilde{\mathcal{F} \mathcal{G}}$ and $(F, G, E) \tilde{\subseteq}\left(F_{1}, G_{1}, E\right) \Longrightarrow\left(F_{1}, G_{1}, E\right) \in \widetilde{\mathcal{F G}}$.

Example 3.2. The family $\widetilde{\mathcal{F G}}=\{(\tilde{X}, \Phi, E)\}$ is a bipolar soft filter over $X$.

Example 3.3. Let $(\Phi, \tilde{X}, E) \neq(F, G, E) \in B S(X, E, \neg E)$. Later, the family $\widetilde{\mathcal{F G}}_{(F, G, E)}=\left\{\left(F_{1}, G_{1}, E\right):(F, G, E) \tilde{\subseteq}\left(F_{1}, G_{1}, E\right) \in B S(X, E, \neg E)\right\}$ is a bipolar soft filter over $X$ and it is called atomic bipolar soft filter.

Example 3.4. Let $E$ be a finite set and $\mathbb{N}$ be the natural numbers. Think the family $\widetilde{\mathcal{F G}}=\left\{(F, G, E): \cup_{e \in E}(\mathbb{N} \times \mathbb{N} \backslash(F(e), G(\neg e)))\right.$ is finite $\}$ is a bipolar soft filter and it is called bipolar soft Frechet filter.

Example 3.5. Let $E$ be a finite set and $X$ be an infinite set. Think the family $\widetilde{\mathcal{F G}}=\left\{(F, G, E): \cup_{e \in E}(X \times X \backslash(F(e), G(\neg e)))\right.$ is finite $\}$ is a bipolar soft filter and it is called bipolar soft cofinite filter.

Example 3.6. Let $E$ be a countable set and $X$ be an uncountable set. Think the family $\widetilde{\mathcal{F G}}=\left\{(F, G, E): \cup_{e \in E}(X \times X \backslash(F(e), G(\neg e)))\right.$ is countable $\}$ is a bipolar soft filter and it is called bipolar soft cocountable filter. 
Proposition 3.7. Let $\widetilde{\mathcal{F G}}$ be a bipoar soft filter over $X$. Later the family

$$
\begin{aligned}
& \widetilde{\mathcal{F}}_{e}=\{F(e):(F, G, E) \in \widetilde{\mathcal{F} \mathcal{G}}\} \\
& \widetilde{\mathcal{G}}_{\neg e}=\{G(\neg e):(F, G, E) \in \widetilde{\mathcal{F} \mathcal{G}}\}
\end{aligned}
$$

for each $e \in E$, do not always define a filter over $X$.

Proof. If $F(e)=\emptyset(G(\neg e)=\emptyset)$ for any $e \in E(\neg e \in \neg E)$ then $\emptyset \in \widetilde{\mathcal{F}}_{e}\left(\emptyset \in \widetilde{\mathcal{G}}_{\neg e}\right)$. Therefore $\widetilde{\mathcal{F}}_{e}\left(\widetilde{\mathcal{G}}_{\neg e}\right)$ is not a filter over $X$.

Example 3.8. Let $X=\left\{x_{1}, x_{2}, x_{3}\right\}, E=\left\{e_{1}, e_{2}, e_{3}\right\}$ and $\widetilde{\mathcal{F G}}=\left\{\left(F_{1}, G_{1}, E\right)\right.$, $\left.\left(F_{2}, G_{2}, E\right),\left(F_{3}, G_{3}, E\right)\right\}$ be a bipolar soft flter defined over $X$ where

$$
\begin{aligned}
& \left(F_{1}, G_{1}, E\right)=\left\{\left(e_{1}, \emptyset,\left\{x_{1}\right\}\right),\left(e_{2},\left\{x_{2}, x_{3}\right\},\left\{x_{2}\right\}\right),\left(e_{3},\left\{x_{1}, x_{2}\right\},\left\{x_{1}\right\}\right)\right\}, \\
& \left(F_{2}, G_{2}, E\right)=\left\{\left(e_{1},\left\{x_{1}, x_{2}\right\},\left\{x_{1}, x_{3}\right\}\right),\left(e_{2},\left\{x_{3}\right\},\left\{x_{2}\right\}\right),\left(e_{3},\left\{x_{2}\right\},\left\{x_{1}\right\}\right)\right\}, \\
& \left(F_{3}, G_{3}, E\right)=\left\{\left(e_{1}, \emptyset,\left\{x_{1}, x_{3}\right\}\right),\left(e_{2},\left\{x_{3}\right\}, \emptyset\right),\left(e_{3},\left\{x_{2}\right\},\left\{x_{1}\right\}\right)\right\} .
\end{aligned}
$$

It can be easily seen that

$$
\widetilde{\mathcal{F}}_{e_{1}}=\left\{\emptyset,\left\{x_{1}, x_{2}\right\}, \emptyset\right\}
$$

and

$$
\widetilde{\mathcal{G}}_{e_{2}}=\left\{\left\{x_{2}\right\},\left\{x_{2}\right\}, \emptyset\right\}
$$

are not filters over $X$.

Definition 3.9. Let's get $\widetilde{\mathcal{F G}}$ and $\widetilde{\mathcal{F}_{1} \mathcal{G}_{1}}$ bipolar soft filters on $X$. If $\widetilde{\mathcal{F G}} \tilde{\subseteq} \widetilde{\mathcal{F}_{1} \mathcal{G}_{1}}$,

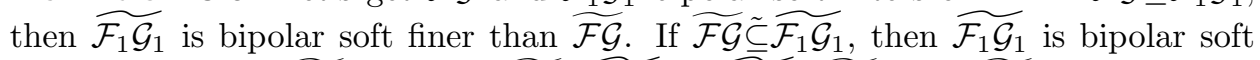
strictly finer than $\widetilde{\mathcal{F G}}$. If either $\widetilde{\mathcal{F} G} \tilde{\mathcal{F}_{1} \mathcal{G}_{1}}$ or $\overline{\mathcal{F}_{1} \mathcal{G}_{1}} \tilde{\subseteq} \widetilde{\mathcal{F G}}$, then $\overline{\mathcal{F G}}$ is comparable with $\widetilde{\mathcal{F}_{1} \mathcal{G}_{1}}$.

Theorem 3.10. Let's take $\left(\widetilde{\mathcal{F}_{i} \mathcal{G}_{i}}\right)_{i \in I}$ as a family of bipolar soft filters over $X$. Later $\widetilde{\mathcal{F G}}=\widetilde{\cap}_{i \in I} \widetilde{\mathcal{F}_{i} \mathcal{G}_{i}}$ is a bipolar soft filter over $X$.

Proof. .

$\left.f g_{1}\right)(\Phi, \tilde{X}, E)$ does not belong to $\widetilde{\mathcal{F G}}=\widetilde{\cap}_{i \in I}\left(\widetilde{\mathcal{F}_{i} \mathcal{G}_{i}}\right)$, because $(\Phi, \tilde{X}, E) \notin \widetilde{\mathcal{F}_{i} \mathcal{G}_{i}}$ for each $i \in I$.

$\left.f g_{2}\right) \operatorname{Let}(F, G, E),\left(F_{1}, G_{1}, E\right) \in \widetilde{\mathcal{F G}}=\widetilde{\cap}_{i \in I} \widetilde{\mathcal{F}_{i} \mathcal{G}_{i}}$ and $(F, G, E),\left(F_{1}, G_{1}, E\right) \in$ $\widetilde{\mathcal{F}_{i} \mathcal{G}_{i}}$ for each $i \in I$. Since $(F, G, E) \tilde{\subseteq}\left(F_{1}, G_{1}, E\right) \in \widetilde{\mathcal{F}_{i} \mathcal{G}_{i}}$ for each $i \in I$, so we obtain $(F, G, E) \tilde{\subseteq}\left(F_{1}, G_{1}, E\right) \in \widetilde{\mathcal{F G}}=\tilde{\cap}_{i \in I} \widetilde{\mathcal{F}_{i} \mathcal{G}_{i}}$.

$\left.f g_{3}\right) \operatorname{Let}(F, G, E) \in \widetilde{\mathcal{F G}}=\tilde{\cap}_{i \in I} \widetilde{\mathcal{F}_{i}} \mathcal{G}_{i}$ and $(F, G, E) \tilde{\subseteq}\left(F_{1}, G_{1}, E\right)$. Since $(F, G, E) \in$ $\widetilde{\mathcal{F}_{i} \mathcal{G}_{i}}$ for each $i \in I$ and $(F, G, E) \subseteq \tilde{\subseteq}\left(F_{1}, G_{1}, E\right)$, we get $\left(F_{1}, G_{1}, E\right) \in \widetilde{\mathcal{F}_{i} \mathcal{G}_{i}}$ for each $i \in I$. Therefore $\left(F_{1}, G_{1}, E\right) \in \widehat{\mathcal{F G}}=\tilde{\cap}_{i \in I} \widetilde{\mathcal{F}_{i} \mathcal{G}_{i}}$.

Remark 3.11. The bipolar soft filter $\widetilde{\mathcal{F G}}$ in Theorem 3.10 is called the greatest lower bound of the family $\left\{\widetilde{\mathcal{F}}_{i} \mathcal{G}_{i}\right\}_{i \in I}$.

Remark 3.12. Generally, the union of bipolar soft filters over $X$ is not a bipolar soft filter over $X$. 
Proposition 3.13. Let's get a bipolar soft family expressed as $\widetilde{\mathcal{S}} \tilde{\subseteq} B S(X, E, \neg E)$. Later there exists a bipolar soft filter $\widetilde{\mathcal{F} \mathcal{G}}$ which contains the bipolar soft family $\widetilde{\mathcal{S}}$, if $\widetilde{\mathcal{S}}$ has this feature: "The all finite intersections of bipolar soft sets of $\widetilde{\mathcal{S}}$ are not bipolar soft empty".

Proof. .

Let's take the $\widetilde{\mathcal{S}}=\left\{\left(F_{i}, G_{i}, E\right): \forall i \in J(J\right.$ is finite $\left.), \tilde{\cap}_{i \in J}\left(F_{i}, G_{i}, E\right) \neq \Phi\right\}$ and take the family that consists of the finite intersections of elements of $\widetilde{\mathcal{S}}$;

$\widetilde{\beta}=\left\{\left(F_{1}, G_{1}, E\right): \forall i \in J(J\right.$ is finite $),\left(F_{i}, G_{i}, E\right) \in \widetilde{\mathcal{S}}$ and $\left.\left(F_{1}, G_{1}, E\right)=\tilde{\cap}_{i \in J}\left(F_{i}, G_{i}, E\right)\right\}$.

Now, let's show that the $\widetilde{\mathcal{F G}}(\widetilde{\mathcal{S}})=\left\{(K, L, E):\left(F_{1}, G_{1}, E\right) \in \widetilde{\beta}\right.$ and $\left(F_{1}, G_{1}, E\right) \sqsubseteq$ $(K, L, E)\}$ family is a bipolar soft filter over $X$.

$\left.f g_{1}\right)$ Since $(\Phi, \tilde{X}, E) \notin \widetilde{\beta}$, for every $(K, L, E) \in \widetilde{\mathcal{F} \mathcal{G}}(\widetilde{\mathcal{S}}),(K, L, E) \neq \Phi$ and so $\Phi \notin \widetilde{\mathcal{F G}}(\widetilde{\mathcal{S}})$.

$\left.f g_{2}\right)$ Let $\left(K_{1}, L_{1}, E\right),\left(K_{2}, L_{2}, E\right) \in \widetilde{\mathcal{F} \mathcal{G}}(\widetilde{\mathcal{S}})$. There exist bipolar soft sets $\left(F_{1}, G_{1}, E\right)$, $\left(F_{2}, G_{2}, E\right) \in \widetilde{\beta}$ such that $\left(F_{1}, G_{1}, E\right) \tilde{\subseteq}\left(K_{1}, L_{1}, E\right)$ and $\left(F_{2}, G_{2}, E\right) \tilde{\subseteq}\left(K_{2}, L_{2}, E\right)$. Using the given definition of $\widetilde{\beta},(\Phi, \tilde{X}, E) \neq\left(F_{1}, G_{1}, E\right)_{1} \tilde{\cap}\left(F_{2}, G_{2}, E\right) \tilde{\subseteq}\left(K_{1}, L_{1}, E\right) \tilde{\cap}\left(K_{2}, L_{2}, E\right)$,

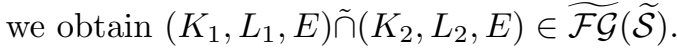

$\left.f g_{3}\right)$ Let $\left(K_{1}, L_{1}, E\right) \in \widetilde{\mathcal{F} \mathcal{G}}(\widetilde{\mathcal{S}})$ and $\left(K_{1}, L_{1}, E\right) \subseteq \tilde{\subseteq}\left(K_{2}, L_{2}, E\right)$. Later, there exists a bipolar soft set $\left(F_{1}, G_{1}, E\right) \in \widetilde{\beta}$ such that $\left(F_{1}, G_{1}, E\right) \tilde{\subseteq}\left(K_{1}, L_{1}, E\right)$. Since

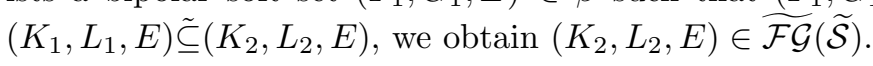

Remark 3.14. The bipolar soft filter $\widetilde{\mathcal{F G}}(\widetilde{\mathcal{S}})$ in Proposition 3.13 is said to be generated by $\widetilde{\mathcal{S}}$ and $\widetilde{\mathcal{S}}$ is said to be bipolar soft filter subbase of $\widetilde{\mathcal{F} \mathcal{G}}(\widetilde{\mathcal{S}})$. Obviously, it is $\widetilde{\mathcal{S}} \tilde{\subseteq} \widetilde{\mathcal{F}}(\widetilde{\mathcal{S}})$.

Proposition 3.15. The bipolar soft filter $\widetilde{\mathcal{F G}}(\widetilde{\mathcal{S}})$ which is generated by $\widetilde{\mathcal{S}}$ is the coarsest bipolar soft filter which contains $\widetilde{\mathcal{S}}$.

Proof. Assume that $\widetilde{\mathcal{S}} \tilde{\subseteq} \widetilde{\mathcal{F}_{1} \mathcal{G}_{1}}$. By Remark 3.14, for every $(K, L, E) \in \widetilde{\mathcal{F G}}(\widetilde{\mathcal{S}})$, there exists a $\left(F_{1}, G_{1}, E\right) \in \widetilde{\beta}$ such that $\left(F_{1}, G_{1}, E\right) \tilde{\subseteq}(K, L, E)$. Since $\widetilde{\beta} \tilde{\subseteq} \widetilde{\mathcal{F}_{1} \mathcal{G}_{1}}$ then $\left(F_{1}, G_{1}, E\right) \tilde{\subseteq} \widetilde{\mathcal{F}_{1} \mathcal{G}_{1}}$. Since $\widetilde{\mathcal{F}_{1} \mathcal{G}_{1}}$ is a bipolar soft filter, $(K, L, E) \in \widetilde{\mathcal{F}_{1} \mathcal{G}_{1}}$ by $f g_{3}$ in Definition 3.1. This way, $\widehat{\mathcal{F} \mathcal{G}}(\widetilde{\mathcal{S}}) \tilde{\subseteq} \widetilde{\mathcal{F}_{1} \mathcal{G}_{1}}$ is achieved.

Theorem 3.16. The family $\left(\widetilde{\mathcal{F G}}_{i}\right)_{i \in I}$ of bipolar soft filters over $X$ has a least upper bound if and only if for all finite subfamilies $\left(\widetilde{\mathcal{F} \mathcal{G}_{i}}\right)_{1 \leq i \leq n}$ of $\left(\widetilde{\mathcal{F} \mathcal{G}_{i}}\right)_{i \in I}$ and all $\left(F_{i}, G_{i}, E\right) \in\left(\widetilde{\mathcal{F} \mathcal{G}_{i}}\right)_{i \in I}(1 \leq i \leq n)$, the intersection $\left(F_{1}, G_{1}, E\right) \widetilde{\cap} \ldots \widetilde{\cap}\left(F_{n}, G_{n}, E\right)$ is not bipolar soft empty.

Proof. $\Longrightarrow$ If there exists a least upper bound of the family $\left(\widetilde{\mathcal{F} \mathcal{G}_{i}}\right)_{i \in I}$, by $f g_{1}$ and $f g_{2}$ in Definition 3.1 , for all finite subfamilies $\left(\widetilde{\mathcal{F} \mathcal{G}_{i}}\right)_{1 \leq i \leq n}$ of $\left(\widetilde{\mathcal{F} \mathcal{G}_{i}}\right)_{i \in I}$ and all $\left(F_{i}, G_{i}, E\right) \in \widetilde{\mathcal{F} \mathcal{G}_{i}}(1 \leq i \leq n)$, the intersection $\left(F_{1}, G_{1}, E\right) \widetilde{\cap} \ldots \widetilde{\cap}\left(F_{n}, G_{n}, E\right) \neq$ $(\Phi, \tilde{X}, E)$.

$\Longleftarrow \operatorname{Let}\left(F_{1}, G_{1}, E\right) \widetilde{\cap} \ldots \widetilde{\cap}\left(F_{n}, G_{n}, E\right) \neq(\Phi, \tilde{X}, E)$ for all finite subfamilies $\left(\widetilde{\mathcal{F} \mathcal{G}_{i}}\right)_{1 \leq i \leq n}$ of $\left(\widetilde{\mathcal{F} \mathcal{G}_{i}}\right)_{i \in I}$ and all $\left(F_{i}, G_{i}, E\right) \in \widetilde{\mathcal{F G}_{i}}(1 \leq i \leq n)$. Then, the bipolar soft filter 
$\widetilde{\mathcal{F} \mathcal{G}}(\widetilde{\mathcal{S}})$ generated by $\widetilde{\mathcal{S}}=\tilde{\cup}_{i \in I} \widetilde{\mathcal{F} \mathcal{G}_{i}}=\left\{(F, G, E):(\exists i \in I)(F, G, E) \in \widetilde{\mathcal{F} \mathcal{G}_{i}}\right\}$ is the least upper bound of the family $\left(\widetilde{\mathcal{F G}}_{i}\right)_{i \in I}$ by Proposition 3.15 .

Definition 3.17. Let $\widetilde{\beta} \tilde{\subseteq} B S(X, E, \neg E)$ be a bipolar soft filter base on $X$ if i) $\widetilde{\beta} \neq(\Phi, \widetilde{X}, E)$ and $(\Phi, \widetilde{X}, E) \notin \widetilde{\beta}$, ii) $\forall(F, G, E),\left(F_{1}, G_{1}, E\right) \in \widetilde{\beta}, \exists(K, L, E) \in \widetilde{\beta} \ni(K, L, E) \tilde{\subseteq}(F, G, E) \tilde{\cap}\left(F_{1}, G_{1}, E\right)$.

Remark 3.18. $\widetilde{\beta}$ which is in Proposition 3.13 is a bipolar soft filter base.

Remark 3.19. It is clear that, every bipolar soft filter is a bipolar soft filter base.

Example 3.20. Let $(\Phi, \widetilde{X}, E) \neq(F, G, E) \in B S(X, E, \neg E)$. Then, the family $\widetilde{\beta}=\{(F, G, E)\}$ is a bipolar soft filter base over $X$.

\section{Conclusion}

The main purpose of this study is to identify the bipolar soft filters given on the initial universe with a fixed set of parameters and to investigate some basic properties. In addition, examples have been presented so that the subject can be better understood. We believe that the results of this study may be useful for the future work of many researchers.

\section{REFERENCES}

[1] A. Aygünoğlu, H. Aygün, Some notes on soft topological spaces, Neural Comput. Appl., Vol. 21, N. 1, pp. 113-119, (2012).

[2] N. Çă̆man, S. Karataş, S. Enginoğlu , Soft topology, Comput. Math. Appl., Vol. 62, pp. 351-358, (2011).

[3] S. Hussain, B. Ahmad, Some properties of soft topological spaces, Comput. Math. Appl., Vol. 62, pp. 4058-4067, (2011).

[4] F. Karaaslan and S. Karatas, A new approach to bipolar soft sets and its applications, Discrete Math. Algorithm. Appl., 07, 1550054, (2015).

[5] P.K. Maji, R. Biswas, and A.R. Roy, Soft set theory, Computers and Mathematics with Applications, Vol. 45 N. 4-5, pp. 555-562, (2003).

[6] W.K. Min, A note on soft topological spaces, Comput. Math. Appl., Vol. 62, pp. 3524-3528, (2011).

[7] D. Molodtsov, Soft set theory first results, Comput. Math. Appl., Vol. 37, pp. 19-31, (1999).

[8] B. Pazar Varol, H. Aygün, On soft hausdorff spaces, Ann. Fuzzy Math. Inf., Vol. 5, N. 1, pp. 15-24, (2013).

[9] M. Shabir, M. Naz, On soft topological spaces, Comput. Math. Appl., 61, 1786-1799, (2011).

[10] M. Shabir and M. Naz, On Bipolar Soft Sets, arXiv: 1303.1344v1 [math.LO], (2013).

[11] M. Shabir and A. Bakhtawar, Bipolar soft connected, bipolar soft disconnected and bipolar soft compact spaces, Songklanakari J. Sci. Technol., Vol. 39, N. 3, pp. 359-371, (2017).

[12] Ş. Yüksel, N. Tozlu, G.Z. Ergül, Soft Filter, Math Sci., Vol. 8, N. 119, (2014).

[13] İ. Zorlutuna, M. Akdağ, W.K. Min, S. Atmaca, Remarks On soft topological spaces, Ann. Fuzzy Math. Inf., Vol. 3, N. 2, pp. 171-185, (2012).

[14] Y.T. Öztürk, On Bipolar Soft Topological Space, Journal of New Theory, Vol. 20, pp. 64-75, (2018).

(author one) Mersin University, Department of Mathematics, Mersin, Turkey.

Current address: Mersin University, Department of Mathematics, Mersin, Turkey.

Email address, author one: orhandlk952495@hotmail.com

(author two) Mersin University, Department of Mathematics, Mersin, Turkey.

Email address, author two: naimedemirtas@mersin.edu.tr 